\title{
Radiation and Inclined Magnetic Field Effects on Unsteady Hydromagnetic Free Convection Flow past an Impulsively Moving Vertical Plate in a Porous Medium
}

\author{
N. Sandeep ${ }^{1 \dagger}$ and V. Sugunamma ${ }^{2}$ \\ ${ }^{1}$ Assistant Professor, Department of Mathematics, SAS, Vellore Institute of Technology, Vellore, India \\ ${ }^{2}$ Associate Professor, Department of mathematics, S.V.University, Tirupat,India
}

$\dagger$ Corresponding Author Email: nsreddy.dr@gmail.com

(Received Febraury 25, 2012; accepted July 9, 2013)

\begin{abstract}
We analyse the effects of radiation and rotation on unsteady hydromagnetic free convection flow of a viscous incompressible electrically conducting fluid past an impulsively moving vertical plate in a porous medium by applying inclined magnetic field, Under Boussinesq approximation, assuming that the temperature of the plate has a temporarily ramped profile. An exact solution of the governing equations, in dimensionless form is obtained by Laplace transform technique. To compare the results obtained in this case with that of isothermal plate and exact solution of the governing equations are also obtained for isothermal plate and results are discussed graphically in both ramped temperature and isothermal cases.
\end{abstract}

Keywords: MHD, Rotation, Radiation, Inclination, Free convection, Ramped temperature.

\section{INTRODUCTION}

The study of convective heat transfer from a solid body with different geometries embedded in a fluid saturated porous medium has varied and wide applications in many areas of science and engineering such as geothermal reservoirs, drying of porous solids, chemical catalytic reactors, thermal insulators, nuclear waste repositories, heat exchanger devices, enhanced oil and gas recovery, underground energy transport etc.

Keeping the above facts Kumar and Varma (2011) have studied the radiation effects on MHD flow past an impulsively started exponentially accelerated vertical plate with variable temperature in the presence of heat generation. The combined effects of rotation and radiation on MHD flow past an impulsively started vertical plate with variable temperature was studied by Rajput and Kumar (2011). Chebbi and Bouzaiane (2012) discussed the effects of rotation on the passive scalar and kinematic fields of homogeneous sheared turbulence. Jha and Ajibade (2010) have studied the unsteady free convective Couette flow of heat generating/absorbing fluid. The unsteady MHD heat and mass transfer free convection flow of polar fluids past a vertical moving porous plate in a porous medium with heat generation and thermal diffusion has been analyzed by Saxena and
Dubey (2011). The effects of thermal radiation and free convection currents on the unsteady Couette flow between two vertical parallel plates with constant heat flux at one boundary have been studied by Narahari (2010). Effects of hall current and rotation on unsteady MHD couette flow in the presence of an Inclined Magnetic field was analyzed by Seth et al (2012).Vijayalakshmi (2010) have studied Radiation effects on free-convection flow past an impulsively started vertical plate in a rotating fluid. The effect of a uniform transverse magnetic field on the unsteady transient free convection flow of an incompressible viscous electrically conducting fluid between two infinite vertical parallel plates with constant temperature was studied by Rajput and Sahu (2011). Bestman and Adjepong (1988) discussed the magneto hydrodynamic free convection flow, with radiative heat transfer, past an infinite moving plate in rotating incompressible, viscous and optically transparent medium. Arpaci (1968) analysed effects of thermal radiation on the laminar free convection from a heated vertical plate. Sandeep and Sugunamma (2013) analyzed aligned magnetic field and chemical reaction effects on flow past a vertical oscillating plate through porous medium

The present paper deals with the effects of radiation and rotation on unsteady hydromagnetic free convection 
flow of a viscous incompressible electrically conducting fluid past an impulsively moving vertical plate in a porous medium by applying inclined magnetic field, Under Boussinesq approximation, assuming that the temperature of the plate has a temporarily ramped profile. An exact solution of the governing equations, in dimensionless form is obtained by Laplace transform technique. To compare the results obtained in this case with that of isothermal plate and exact solution of the governing equations are also obtained for isothermal plate.

\section{MATHEMATICAL FORMULATION}

We consider an unsteady hydromagnetic free convection flow of a viscous incompressible electrically conducting fluid past an impulsively moving infinite vertical plate embedded in a porous medium. And consider the coordinate system in such a way that the $x$-axis is taken along the plate in the upward direction, $y$-axis normal to the plane of the plate in the fluid and z-axis perpendicular to $x y$ - plane. The fluid is permitted by an inclined magnetic field applied along the direction of $y$ axis. Both the fluid and plate rotate in unison with a permit uniform angular velocity $\Omega$ about $y$-axis. Initially, at time $t^{*} \leq 0$, both the fluid and plate are at rest and at a constant temperature $T_{\infty}^{*}$. At time $t^{*}>0$, the plate starts moving in $x$ direction with uniform velocity $U_{0}$ and the temperature of the plate is raised or lowered to $T_{\infty}^{*}+\left(T_{w}^{*}-T_{\infty}^{*}\right) t * / t_{0}$ when $t^{*} \leq t_{0}$, and thereafter, for $t^{*}>t_{0}$, it is maintained at the constant temperature $T_{w}^{*}$. Since the plate is infinite in $x$ and $z$ directions and is electrically non-conducting with all physical quantities, except pressure will be functions of $y$ and $t^{*}$ only.

Taking into consideration the assumptions made above, the governing equations for laminar free convection flow of a viscous incompressible electrically conducting fluid past a vertical plate in a uniform porous medium with radiative heat transfer, under Boussinesq approximation, in a rotating frame of reference are

$$
\frac{\partial u^{*}}{\partial t^{*}}+2 \Omega w^{*}=v \frac{\partial^{2} u^{*}}{\partial y^{2}}+g \beta^{*}\left(T^{*}-T_{\infty}{ }^{*}\right)-\frac{v}{K_{1}^{*}} u^{*}-B \operatorname{Sin}^{2} \theta u^{*}
$$

$$
\frac{\partial w^{*}}{\partial t^{*}}-2 \Omega u^{*}=v \frac{\partial^{2} w^{*}}{\partial y^{2}}-\frac{v}{K_{1}^{*}} w^{*}-B \operatorname{Sin}^{2} \theta w^{*}
$$

$\frac{\partial T^{*}}{\partial t^{*}}=\frac{k}{\rho c_{p}} \frac{\partial^{2} T^{*}}{\partial y^{2}}-\frac{1}{\rho c_{p}} \frac{\partial q_{r}^{*}}{\partial y}$

where $T^{*}, g, \beta^{*}, v, \sigma, \rho, k, K_{1}^{*}, c_{p}$ and $q_{r}^{*}$ are respectively, temperature of the fluid, acceleration due to gravity, volumetric coefficient of thermal expansion, kinematic coefficient of viscosity, electrical conductivity, fluid density, thermal conductivity, permeability of porous medium, specific heat at constant pressure and radiative flux vector. Here $B=\frac{\sigma B_{0}{ }^{2}}{\rho}$ is applied magnetic field.

The initial and boundary conditions are

$u^{*}=w^{*}=0, T^{*}=T_{\infty}^{*}$ for $y \geq 0$ and $t^{*} \leq 0$,

$u^{*}=U_{0}, w^{*}=0$ at $y=0$ for $t^{*}>0$,

$T^{*}=T_{\infty}^{*}+\left(T_{w}^{*}-T_{\infty}^{*}\right) t^{*} / t_{0}$ at $y=0$ for $\left.0<t^{*} \leq t_{0},\right\}$

$T^{*}=T_{w}^{*}$ at $y=0$ for $t^{*}>t_{0}$,

$u^{*} \rightarrow 0, w^{*} \rightarrow 0, T^{*} \rightarrow T_{\infty}^{*}$ as $y \rightarrow \infty$ for $t^{*}>0$.

We now use Rosseland approximation which leads to the value of radiative heat flux $q_{r}^{*}$ as

$$
q_{r}^{*}=-\frac{4 \sigma^{*}}{3 k^{*}} \frac{\partial T^{* 4}}{\partial y},
$$

Where $k^{*}$ is mean absorption coefficient and $\sigma^{*}$ is Stefan-Boltzmann constant. It may be noted that by using Rosseland approximation we limit our analysis to optically thick fluids. Assuming small temperature differences between fluid temperature $T^{*}$ and free stream temperature $T_{\infty}^{*}$, the Eq. (5) is linearized by expanding $T *^{4}$ in Taylors series about free stream temperature $T_{\infty}^{*}$, after neglecting second and higher order terms in $\left(T^{*}-T_{\infty}^{*}\right)$ it takes the form

$T^{* 4} \cong 4 T_{\infty}^{* 3} T^{*}-3 T_{\infty}^{* 4}$

Making use of Eqs. (5) and (6), Eq. (3) becomes

$\frac{\partial T^{*}}{\partial t^{*}}=\frac{k}{\rho c_{p}} \frac{\partial^{2} T^{*}}{\partial y^{2}}+\frac{1}{\rho c_{p}} \frac{16 \sigma^{*} T_{\infty}^{* 3}}{3 k^{*}} \frac{\partial^{2} T^{*}}{\partial y^{2}}$

Introduce the following non-dimensional variables

$Y=y / U_{0} t_{0}, \boldsymbol{u}=u^{*} / U_{0}, w=w^{*} / U_{0}$,
$t=t^{*} / t_{0}$ and $T=\left(T^{*}-T_{\infty}^{*}\right) /\left(T_{w}^{*}-T_{\infty}^{*}\right)$,

The Eqs. (1), (2) and (3) in the non-dimensional form are 


$$
\frac{\partial u}{\partial t}+2 K^{2} w=\frac{\partial^{2} u}{\partial Y^{2}}+G_{r} T-\left(M+\frac{1}{K_{1}}\right) u
$$

$$
\begin{aligned}
& \frac{\partial w}{\partial t}-2 K^{2} u=\frac{\partial^{2} w}{\partial Y^{2}}-\left(M+\frac{1}{K_{1}}\right) w \\
& \frac{\partial T}{\partial t}=\frac{(1+R)}{P_{r}} \frac{\partial^{2} T}{\partial Y^{2}}
\end{aligned}
$$

Where $K^{2}=\Omega v / U_{0}^{2}$ is rotation parameter, $M^{\prime}=\sigma B_{0}^{2} v / \rho U_{0}^{2} \quad$ is magnetic parameter, $M=M \cdot \sin ^{2} \theta$ is inclined magnetic field. $K_{1}=K_{1}^{\prime} U_{0}^{2} / v^{2}$ Is porosity parameter, $G_{r}=g \beta^{\prime} v\left(T_{w^{\prime}}^{\prime}-T_{\infty}^{\prime}\right) / U_{0}{ }^{3} \quad$ is Grashof number, $P_{r}=\rho v c_{p} / k$ is Prandtl number and $R=16 \sigma^{*} T_{\infty}^{\prime 3} / 3 k k^{*}$ is radiation parameter. According to above non dimensionalization process, the characteristic time $t_{o}$ can be defined as

$$
t_{0}=v / U_{0}^{2} \text {. }
$$

Making use of (12) the initial and boundary conditions (5), in non-dimensional form, reduces to

$$
\begin{aligned}
& u=w=0, T=0 \text { for } \mathrm{Y} \geq 0 \text { and } t \leq 0, \\
& u=1, w=0 \text { at } \mathrm{Y}=0 \text { for } t>0 \text {, } \\
& T=t \text { at } \mathrm{Y}=0 \text { for } 0<t \leq 1 \text {, } \\
& T=1 \text { at } \mathrm{Y}=0 \text { for } t>1 \text {, } \\
& u \rightarrow 0, w \rightarrow 0, T \rightarrow 0 \text { as } \mathrm{Y} \rightarrow \infty \text { for } t>0 \text {. }
\end{aligned}
$$

combining (9) and (10) in the form of $f=u+i w$, we obtain

$$
\frac{\partial f}{\partial t}-2 i K^{2} f=\frac{\partial^{2} f}{\partial Y^{2}}-M f-\frac{1}{K_{1}} f+G_{r} T .
$$

The initial and boundary conditions (13) in combined form are

$$
\begin{aligned}
& f=0, T=O \text { for } \mathrm{Y} \geq 0 \text { and } t \leq 0 \\
& f=1 \text { at } \mathrm{Y}=\mathrm{O} \text { for } t>0 \\
& T=t \text { at } \mathrm{Y}=\mathrm{O} \text { for } \mathrm{O}<t \leq 1, \\
& T=1 \text { at } \mathrm{Y}=\mathrm{O} \text { for } t>1,
\end{aligned}
$$

$f \rightarrow \mathrm{O}, T \rightarrow \mathrm{O}$ as $\mathrm{Y} \rightarrow \infty$ for $t>0$.

\section{SOLUTION OF THE PROBLEM}

It is evident from the Eqs. (11) and (14) that the energy Eq. (11) is uncoupled from the Eq. (14). Therefore, first we can obtain the solution for the fluid temperature
$T(Y, t)$ by solving Eq. (11) and then using it in Eq. (14) the solution for $f(Y, t)$ can be obtained.

Applying Laplace transform technique, the Eqs. (11) and (14) with the help of ( 150$)$ reduces to

$$
\frac{d^{2} \tilde{f}}{d Y^{2}}-\left(s+M+\frac{1}{K_{1}}-2 i K^{2}\right) \tilde{f}+G_{r}(\tilde{\overline{1}} \overline{\overline{1}})
$$

$\frac{d^{2} \tilde{T}}{d Y^{2}}-a s \tilde{T}=0$

where $a=P_{r} /(1+R)$ and $\tilde{f}(Y, s)$ and

$\tilde{T}(Y, s)$ are Laplace transforms of $f(Y, t)$ and $T(Y, t)$ respectively defined by

$\tilde{f}(Y, s)=\int_{0}^{\infty} f(Y, t) e^{-s t} d t \quad$ and

$\tilde{T}(Y, s)=\int_{0}^{\infty} T(Y, t) e^{-s t} d t$

( $s>0$ Being Laplace transform parameter).

The boundary conditions (15b) to (15e) becomes

$\tilde{f}=1 / s, \tilde{T}=\left(1-e^{-s}\right) / s^{2}$ at $\left.\mathrm{Y}=0,\right\}$

$\tilde{f} \rightarrow 0, \tilde{T} \rightarrow 0$ as $\mathrm{Y} \rightarrow \infty$.

The solutions of Eqs. (16) and (17), subject to the boundary conditions (18) are given by

$$
\begin{aligned}
& \tilde{T}(Y, s)=\frac{1-e^{-s}}{s^{2}} e^{-Y \sqrt{a s}}, \\
& \tilde{f}(Y, s)=\frac{1}{s} e^{-Y \sqrt{s+\lambda}}+\alpha \frac{(14) 1-e^{-s}}{s^{2}(s-\beta)}\left\{e^{-Y \sqrt{s+\lambda}}-e^{-Y \sqrt{a s}}\right\},
\end{aligned}
$$

where

$$
\left.\begin{array}{l}
\lambda=\left(M+\frac{1}{K_{1}}-2 i K^{2}\right), \\
\alpha=G_{r} /(a-1), \beta=\lambda /(a-1) .
\end{array}\right\}
$$

Taking the inverse Laplace transform of Eqs. (19) and (20), the exact solution for the fluid temperature $T(Y, t)$ and fluid velocity $f(Y, t)=u(Y, t)+i w(Y, t)$ are obtained and expressed in the following form as 


$$
\begin{aligned}
& T(Y, t)=P(Y, t)-H(t-1) P(Y, t-1) \\
& f(Y, t)=\frac{1}{2}\left[\begin{array}{c}
e^{Y \sqrt{\lambda}} \operatorname{erfc}\left(\frac{Y}{2 \sqrt{t}}+\sqrt{\lambda t}\right) \\
+e^{-Y \sqrt{\lambda}} \operatorname{erfc}\left(\frac{Y}{2 \sqrt{t}}-\sqrt{\lambda t}\right)
\end{array}\right]+ \\
& +\alpha[F(Y, t)-H(t-1) F(Y, t-1)],
\end{aligned}
$$

Where

$$
P(Y, t)=\left(\frac{a Y^{2}}{2}+t\right) \operatorname{erfc}\left(\frac{Y \sqrt{a}}{2 \sqrt{t}}\right)-\sqrt{\frac{a t}{\pi}} Y e^{-\frac{a Y^{2}}{4 t}}
$$

$$
\begin{gathered}
F(Y, t)=\frac{e^{\beta t}}{2 \beta^{2}}\left\{\begin{array}{l}
e^{Y \sqrt{\lambda+\beta}} \operatorname{erfc}\left(\frac{Y}{2 \sqrt{t}}+\sqrt{(\lambda+\beta) t}\right) \\
\left.+e^{-Y \sqrt{\lambda+\beta}} \operatorname{erfc}\left(\frac{Y}{2 \sqrt{t}}-\sqrt{(\lambda+\beta) t}\right)\right\}- \\
-\left\{\frac{1}{2 \beta^{2}}+\frac{1}{\beta}\left(\frac{t}{2}+\frac{Y}{4 \sqrt{\lambda}}\right)\right\} e^{Y \sqrt{\lambda}} \operatorname{erfc}\left(\frac{Y}{2 \sqrt{t}}+\sqrt{\lambda t}\right) \\
-\left\{\frac{1}{2 \beta^{2}}+\frac{1}{\beta}\left(\frac{t}{2}-\frac{Y}{4 \sqrt{\lambda}}\right)\right\} e^{-Y \sqrt{\lambda}} \operatorname{erfc}\left(\frac{Y}{2 \sqrt{t}}-\sqrt{\lambda t}\right) \\
-\frac{e^{\beta t}}{2 \beta^{2}}\left\{e^{Y \sqrt{\beta a}} \operatorname{erfc}\left(\frac{Y \sqrt{a}}{2 \sqrt{t}}+\sqrt{\beta t}\right)+e^{-Y \sqrt{\beta a}} \times\right. \\
\left.\operatorname{erfc}\left(\frac{Y \sqrt{a}}{2 \sqrt{t}}-\sqrt{\beta t}\right)\right\}+\frac{1}{\beta^{2}} \operatorname{erfc}\left(\frac{Y \sqrt{a}}{2 \sqrt{t}}\right)+ \\
\frac{1}{\beta}\left\{\left(\frac{a Y^{2}}{2}+t\right) \operatorname{erfc}\left(\frac{Y \sqrt{a}}{2 \sqrt{t}}\right)-\sqrt{\frac{a t}{\pi}} Y e^{-\frac{a Y^{2}}{4 t}}\right\} .
\end{array}\right.
\end{gathered}
$$

In the Eqs. (22) to (25), $\operatorname{erfc}(x)$ is the complementary error function and $\boldsymbol{H}(\boldsymbol{t}-\mathbf{1})$ is the Heaviside unit step function.

\section{SOLUTION IN CASE OF ISOTHERMAL PLATE}

The analytical solution for the fluid temperature and velocity, represented by Eqs.(22) and (23) respectively, are obtained for an unsteady hydromagnetic free convection flow of a viscous incompressible electrically conducting fluid near a vertical moving plate with ramped temperature. In order to highlight the effects of ramped temperature distribution within the plate on the fluid flow, it may be worthwhile to compare such a flow with the one, near a moving plate with uniform temperature. Taking into account the assumptions made in the present study, the solution for the fluid temperature and velocity for the fluid flow near a vertical moving isothermal plate is obtained and expressed as

$T(Y, t)=\operatorname{erfc}\left(\frac{Y}{2} \sqrt{\frac{a}{t}}\right)$

$f(Y, t)=\frac{\left(1-\alpha^{\prime}\right)}{2}\left[\begin{array}{c}e^{Y \sqrt{\lambda}} \operatorname{erfc}\left(\frac{Y}{2 \sqrt{t}}+\sqrt{\lambda t}\right)+ \\ e^{-Y \sqrt{\lambda}} \operatorname{erfc}\left(\frac{Y}{2 \sqrt{t}}-\sqrt{\lambda t}\right)\end{array}\right]+$

$+\frac{\alpha^{\prime} e^{\beta t}}{2}\left[e^{Y \sqrt{\lambda+\beta}} \operatorname{erfc}\left(\frac{Y}{2 \sqrt{t}}+\sqrt{(\lambda+\beta) t}\right)+\right.$

$\left.e^{-Y \sqrt{\lambda+\beta}} \operatorname{erfc}\left(\frac{Y}{2 \sqrt{t}}-\sqrt{(\lambda+\beta) t}\right)\right]+$

$+\alpha^{\prime} \operatorname{erfc}\left(\frac{Y}{2} \sqrt{\frac{a}{t}}\right)$

$-\frac{\alpha^{\prime} e^{\beta t}}{2}\left[e^{Y \sqrt{\beta a}} \operatorname{erfc}\left(\frac{Y}{2} \sqrt{\frac{a}{t}}+\sqrt{\beta t}\right)+\right.$

$\left.e^{-Y \sqrt{\beta a}} \operatorname{erfc}\left(\frac{Y}{2} \sqrt{\frac{a}{t}}-\sqrt{\beta t}\right)\right]$

Where $\alpha^{\prime}=\alpha / \beta$.

\section{RESULTS AND DISCUSSION}

To study the effects of radiation, magnetic field, rotation, porosity of medium, inclined angle and time on the flow-field the numerical values of fluid velocity are displayed graphically versus boundary layer coordinate $\mathrm{Y}$ in Figs.1 to 12 for various values of magnetic parameter M, rotation parameter $K^{2}$, Grashof number $G_{r}$, radiation parameter $R$, porosity parameter $K_{1}$ ,inclined angle $\theta$ and time $t$ taking $P_{r}=0.71$ (ionized air).Here inclined angle is taken as $\theta=\frac{\pi}{2}$ for discussing velocity and temperature profiles.

It is noticed from Fig.1 that an increase in the magnetic parameter $M$ leads to a decrease in the velocity for ramped temperature and isothermal plates. But initially at isothermal plate velocity takes reverse action. i.e. increase in magnetic field causes an increase of velocity after wards velocity decreases gradually in increase magnetic field. This is due to the fact that the application of an inclined magnetic field to an electrically 
N. Sandeep and V. Sugunamma / JAFM, Vol. 7, No. 2, pp. 275-286, 2014.

conducting fluid gives rise to a resistive force which is known as Lorentz force. From Fig. 2 It is evident that, for both ramped temperature and isothermal plates, an increase in rotation parameter $K^{2}$ leads to a decrease in the velocity near the plate. From Fig. 3 it is found that the velocity of the fluid at ramped temperature increases an increase in Grashof number $G_{r}$ but in an isothermal plate, velocity varies in certain time and follows ramped profile. Fig. 4 that the velocity increases by increase in the radiation parameter $\mathrm{R}$,but at ramped temperature velocity decreases with an increase in the radiation. Fig. 5 shows that the effect of the fluid velocity at ramped temperature and an isothermal plates, for different values of porosity parameter $K_{1}$. It is observed that an increase in porosity parameter causes an increase in velocity. It is revealed from Fig.6 that the velocity decreases with an increase in the time at isothermal plate but at ramped temperature, velocity increases by increasing the time $t$.
Figs. 7 and 8 show the effect of inclined magnetic field over velocity. From these it is observed that at ramped temperature, velocity of the fluid decreases by an increase in inclined angle. In the similar manner at isothermal plate also, velocity varies initially and then it is decreased by increase in an inclined angle.

In order to have physical view of fluid temperature, the profiles of fluid temperature are drawn versus boundary layer coordinate $Y$ in Figs. 9 to 12 for various values of radiation parameter $R$, Prandtl number $P_{r}$ and time $t$.It is evident from Fig. 9 that the fluid temperature $T$ increases by an increase in the radiation parameter at ramped temperature but it is reversed in case of isothermal plate. From Fig.10, it is observed that the fluid temperature $T$ increases on increasing Prandtl number $P_{r}$ for both ramped temperature and isothermal plates. It is evident from Figs. 11 and 12 shows that an increase in the time causes the decrease the fluid temperature in both cases.

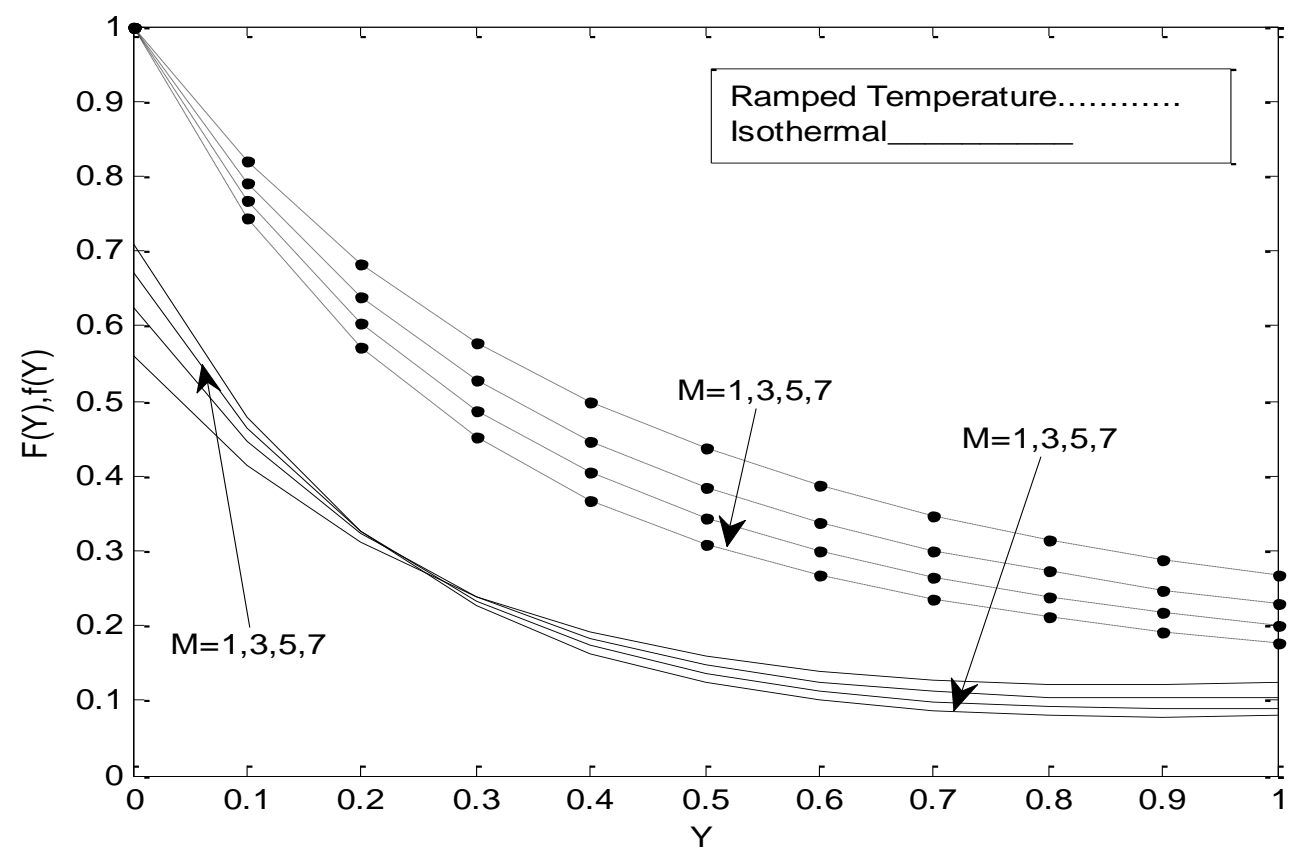

Fig. 1. Velocity field for different values of $M$. When $K^{2}=1, G_{r}=5, R=3, K_{1}=0.1$ and $t=1$ 
N. Sandeep and V. Sugunamma / JAFM, Vol. 7, No. 2, pp. 275-286, 2014.

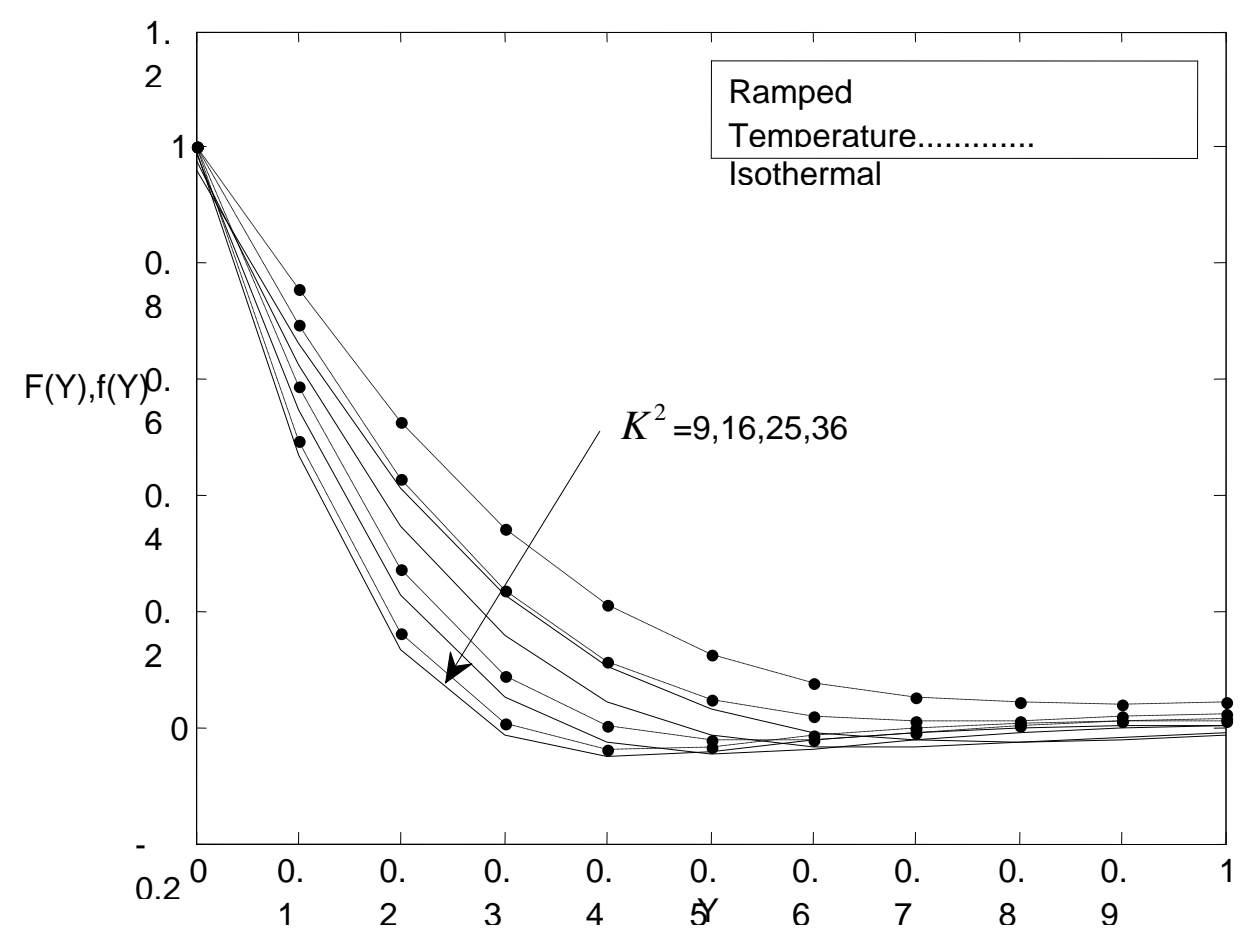

Fig. 2. Velocity field for different values of $K^{2}$. When $M=4, G_{r}=5, R=3, K_{1}=1$ and $t=2$

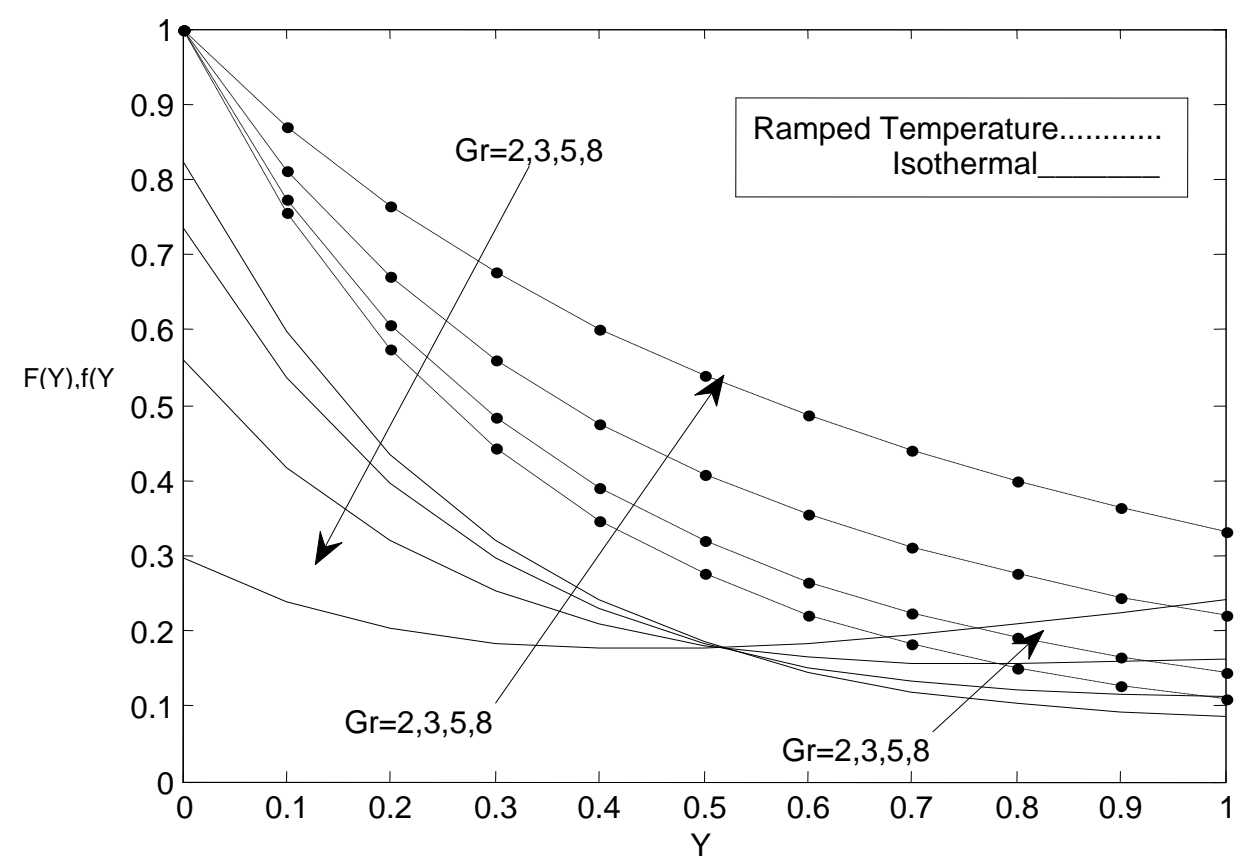

Fig. 3. Velocity field for different values of $G r$. When $K^{2}=1, M=1, R=1, K_{1}=0.1$ and $t=1$ 


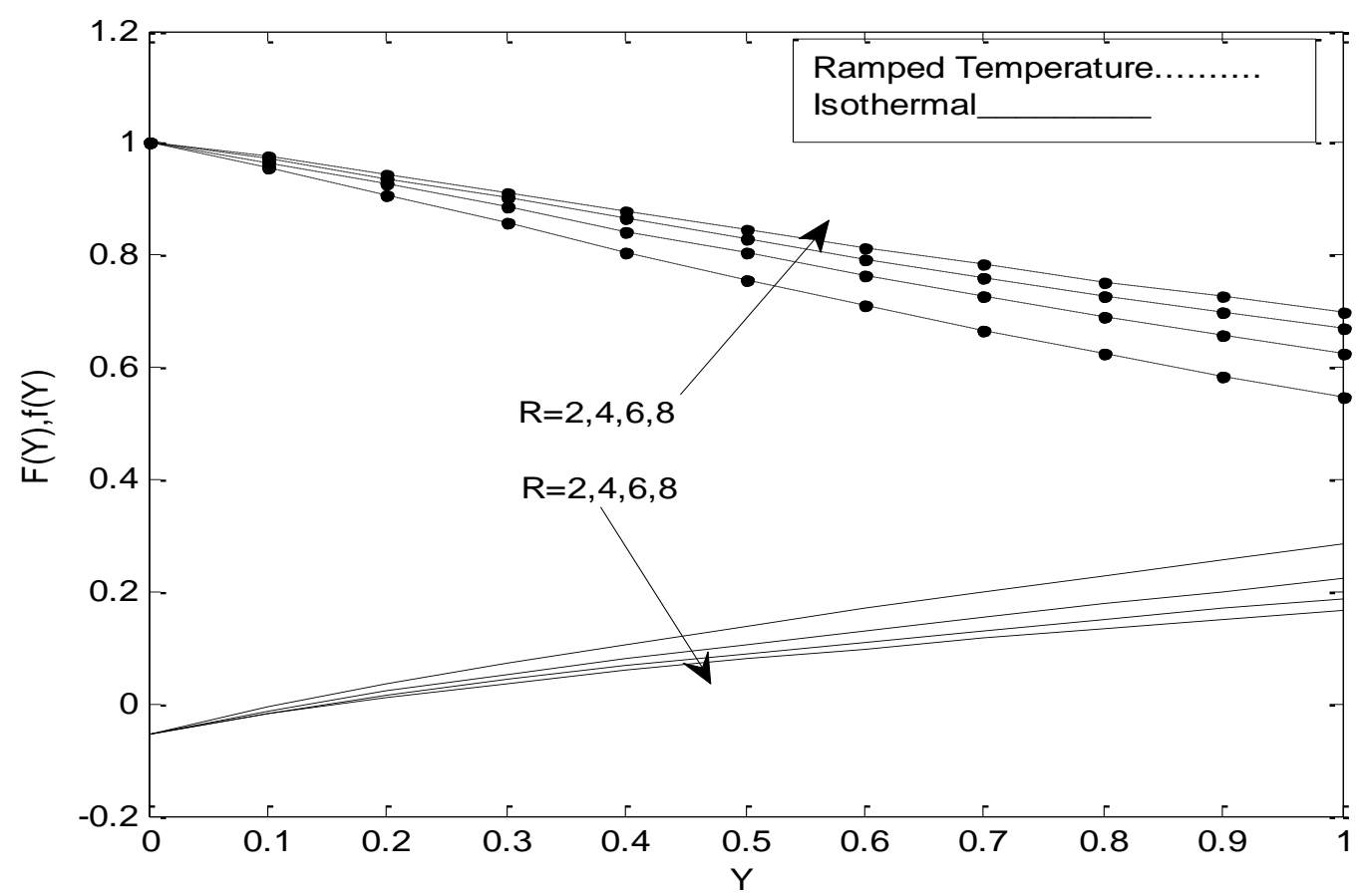

Fig. 4. Velocity field for different values of $R$. When $K^{2}=1, G_{r}=5, M=1, K_{1}=0.1$ and $t=1$

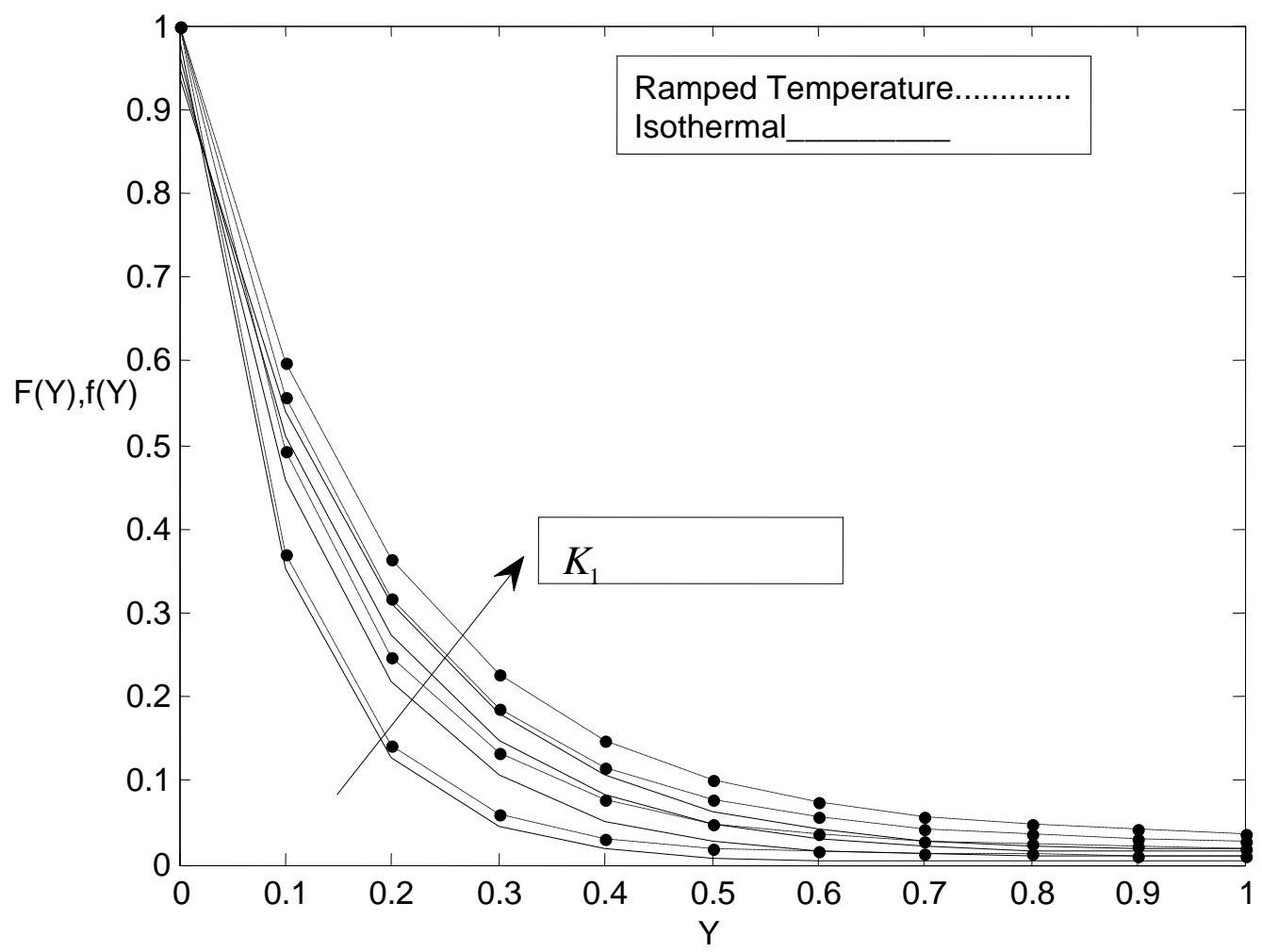

Fig. 5. Velocity field for different values of $K_{1}$. When $K^{2}=4, M=5, R=2, G_{r}=2$ and $t=1$ 


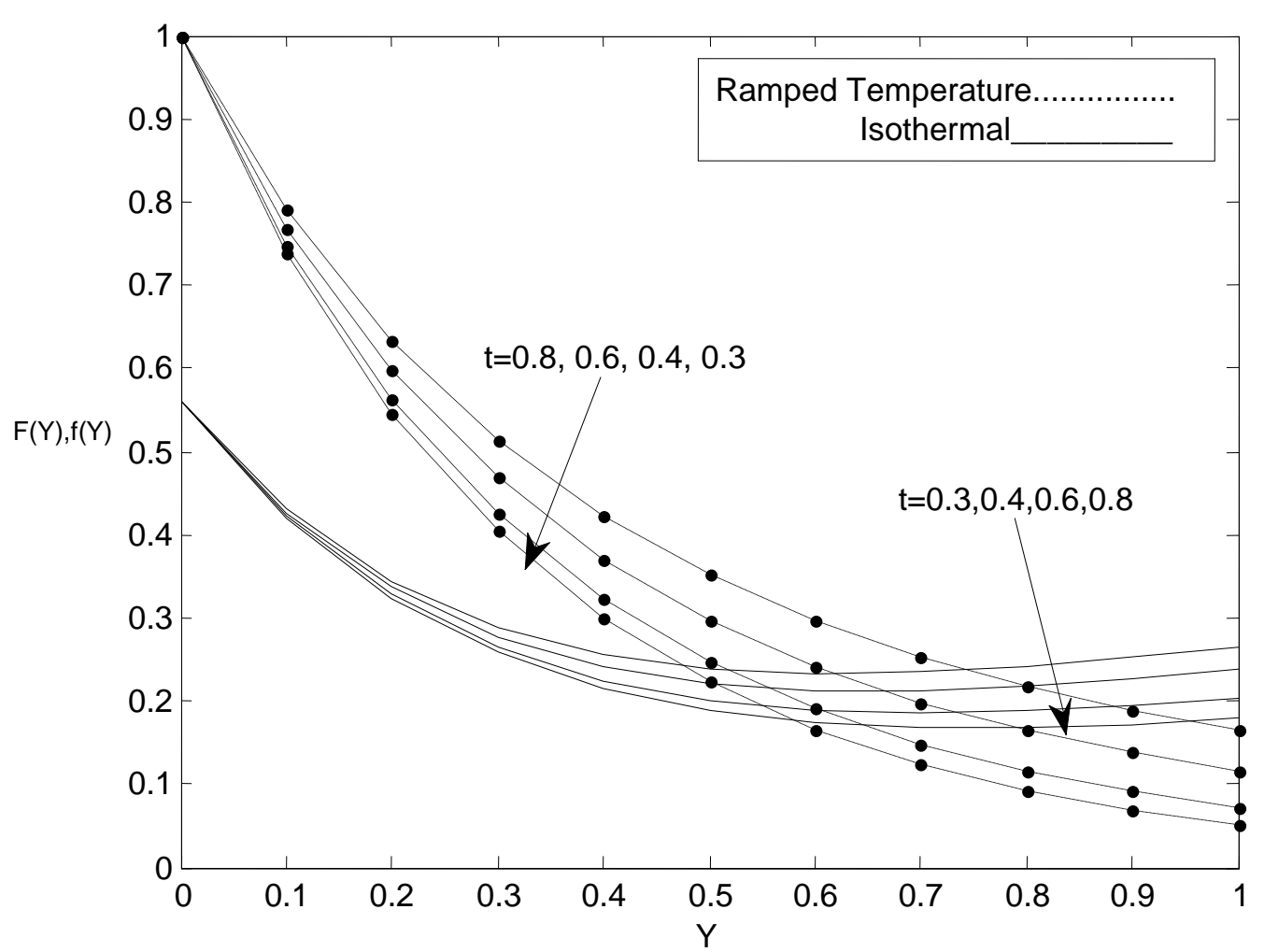

Fig. 6. Velocity field for different values of $t$. When $K^{2}=4, M=1, R=1, K_{1}=0.1$ and $G_{r}=5$

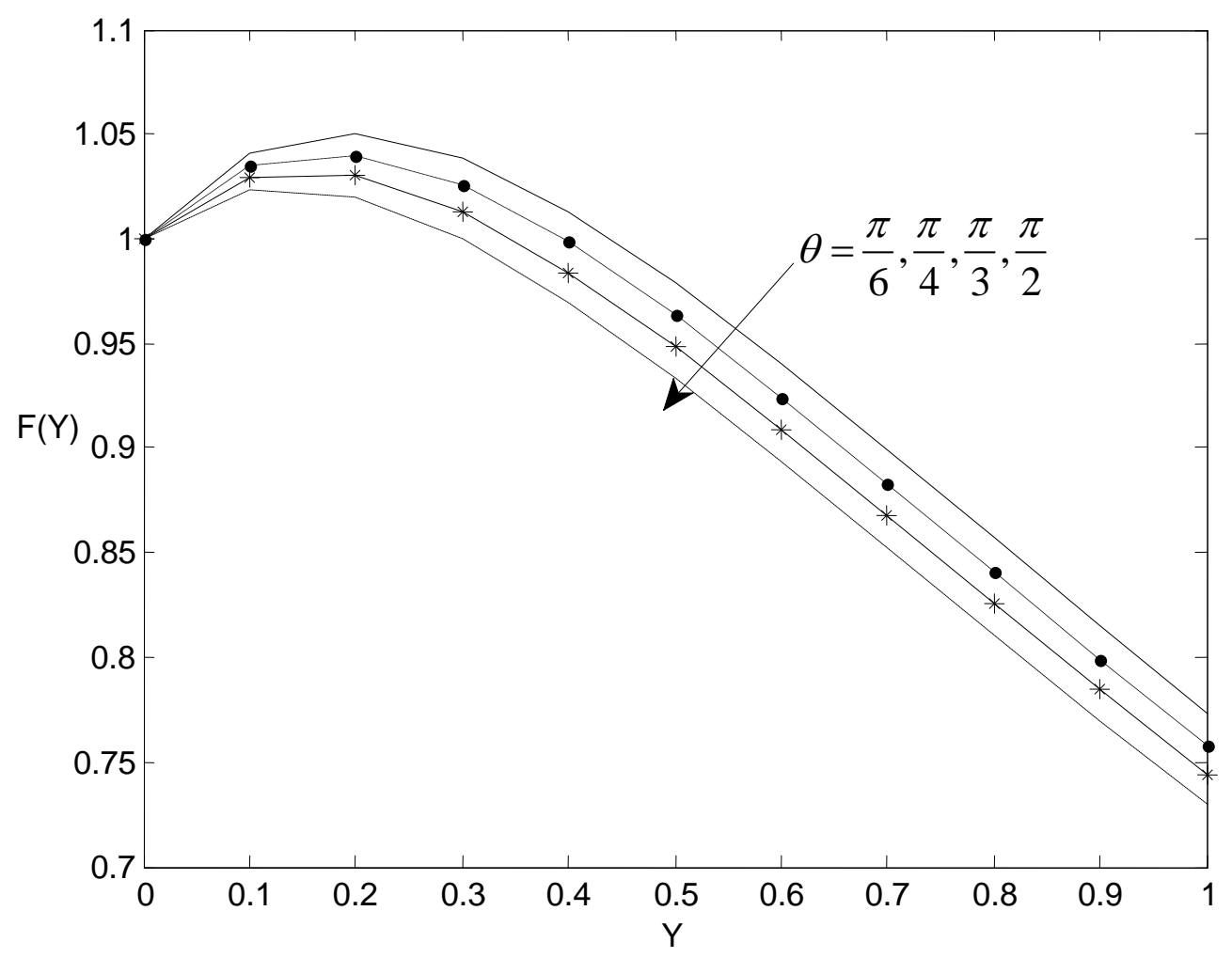

Fig. 7. Velocity field for different values of $\theta$. When $K^{2}=1, M=1, R=3, K_{1}=0.1, G_{r}=15$ 
N. Sandeep and V. Sugunamma / JAFM, Vol. 7, No. 2, pp. 275-286, 2014.

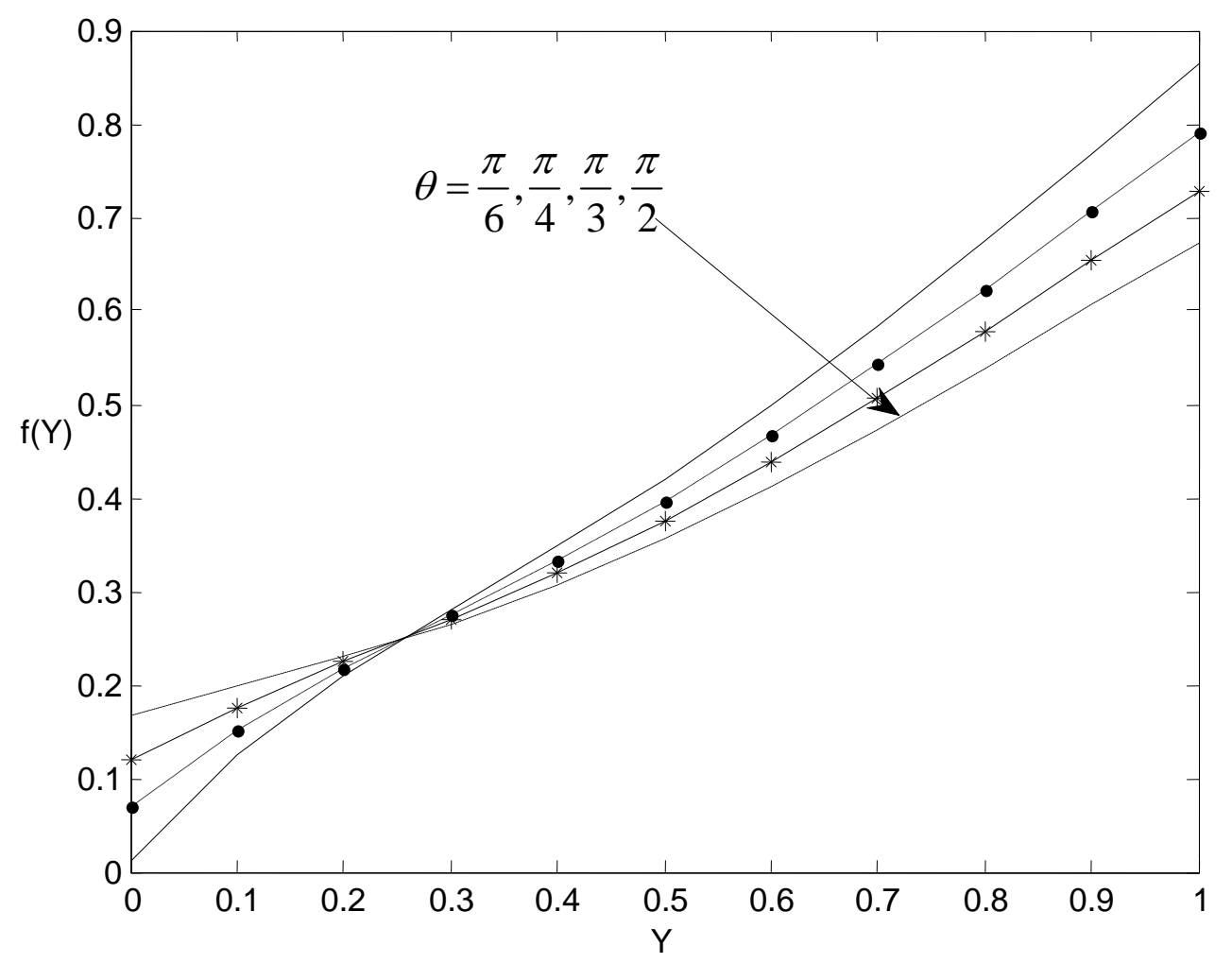

Fig. 8. Velocity field for different values of $\theta$. When $K^{2}=1, M=1, R=3, K_{1}=0.1, G_{r}=15$ and $t=1$.

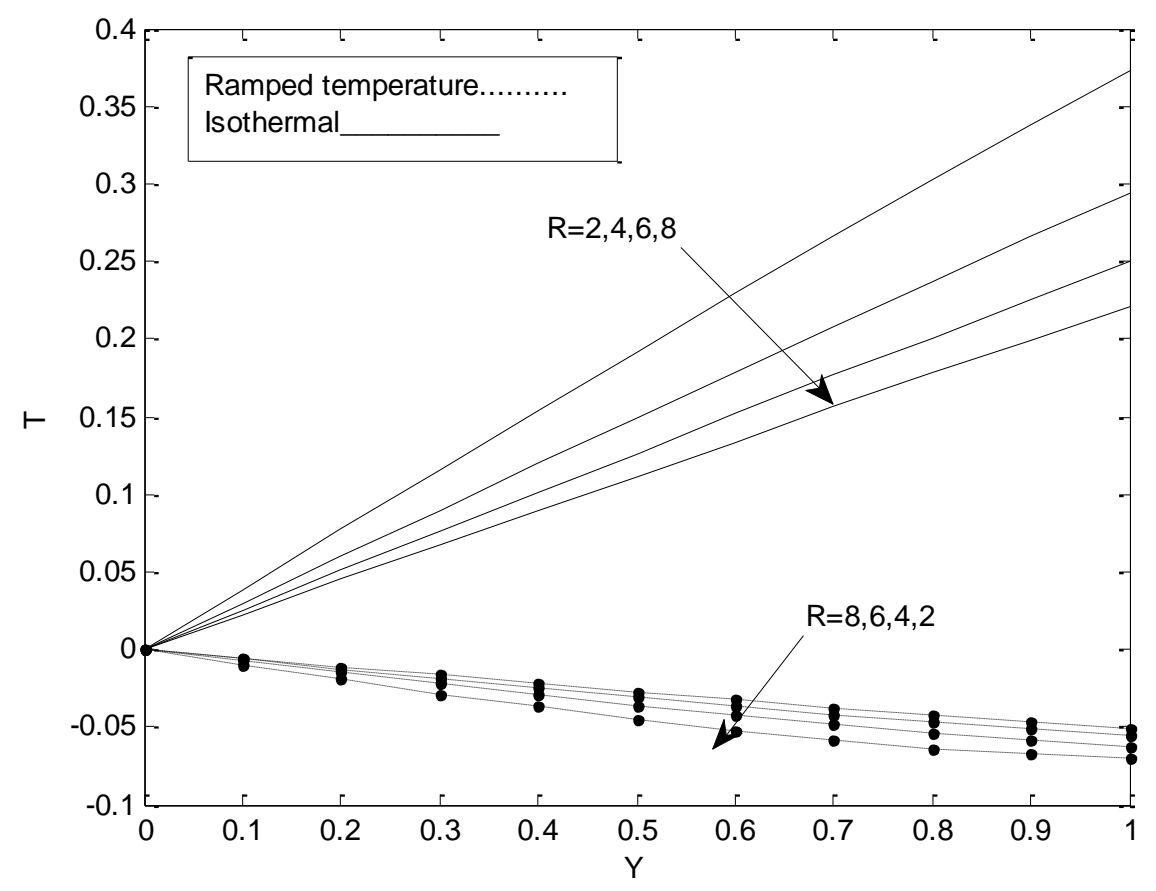

Fig. 9. Temperature field for different values of $R$. When $K 2=4, M=2, R=2, K 1=0.02, G r=3$ and $t=0.5$ 


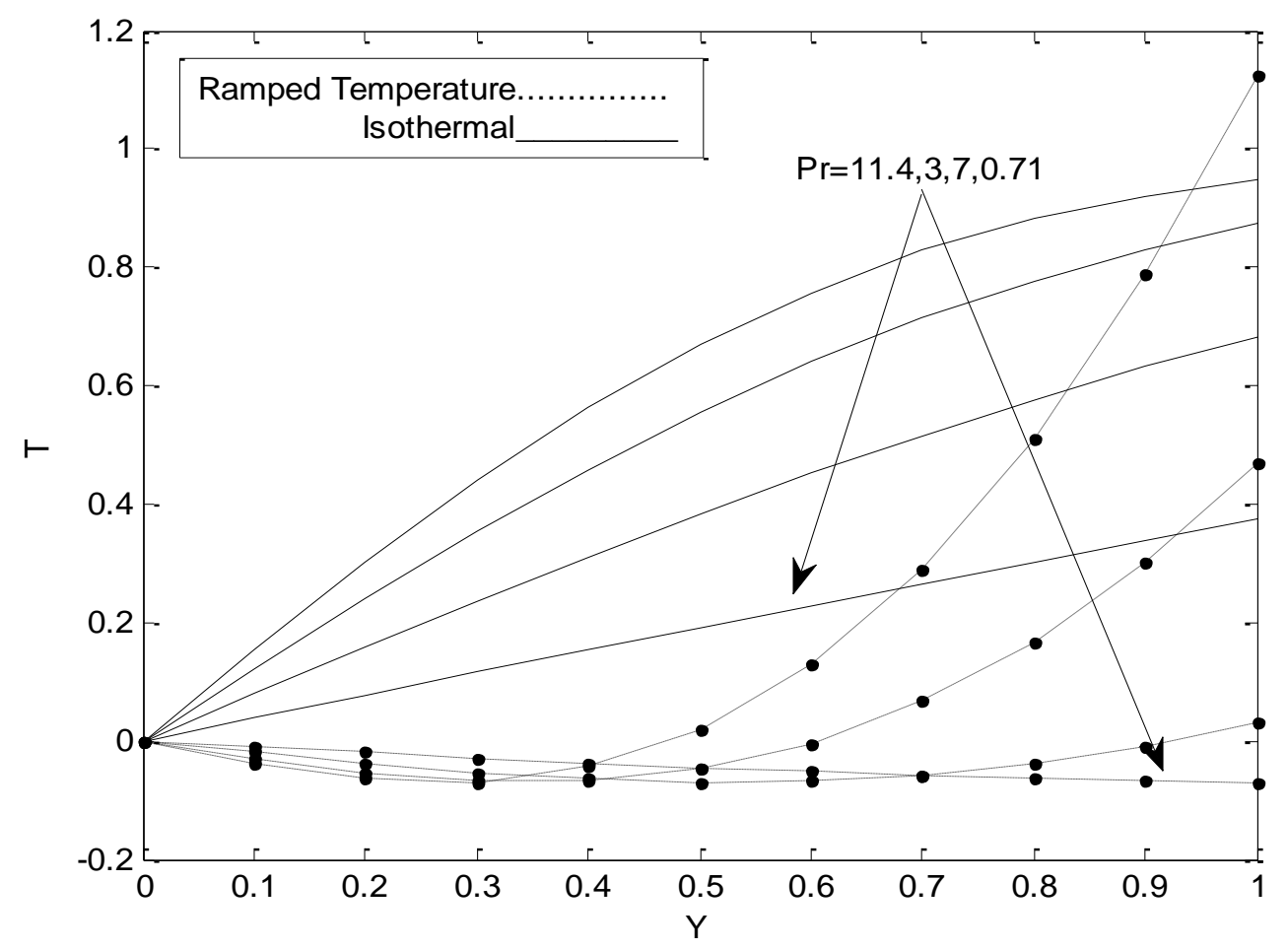

Fig. 10. Temperature field for different values of Pr. When $K^{2}=4, M=2, R=2, K_{1}=0.02, G_{r}=3$ and $t=0.5$

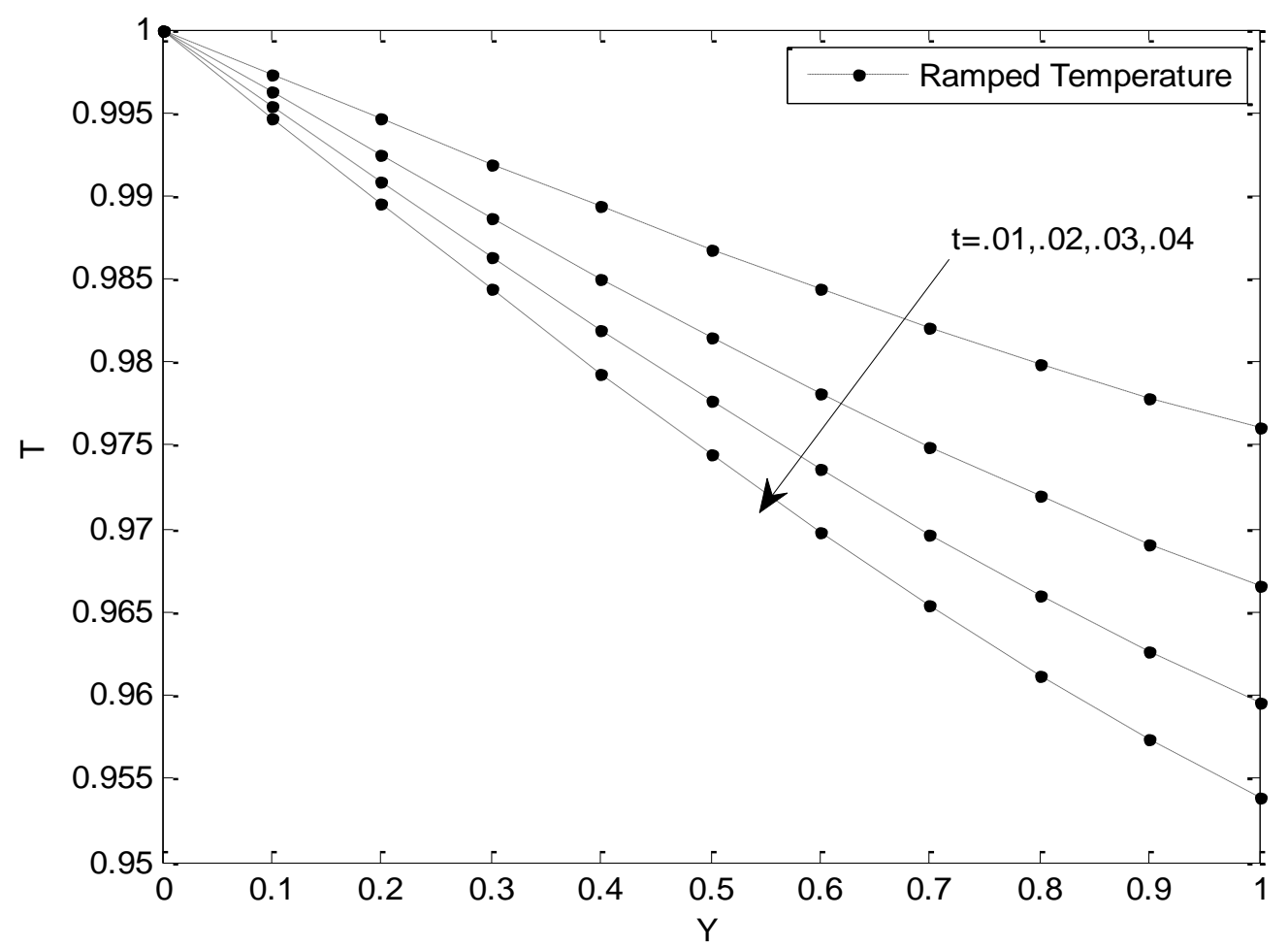

Fig. 11. Temperature field for different values of $t$ in Ramped temperature. When $K^{2}=4, M=2, R=2, K_{1}=0.2, G_{r}=3$ 


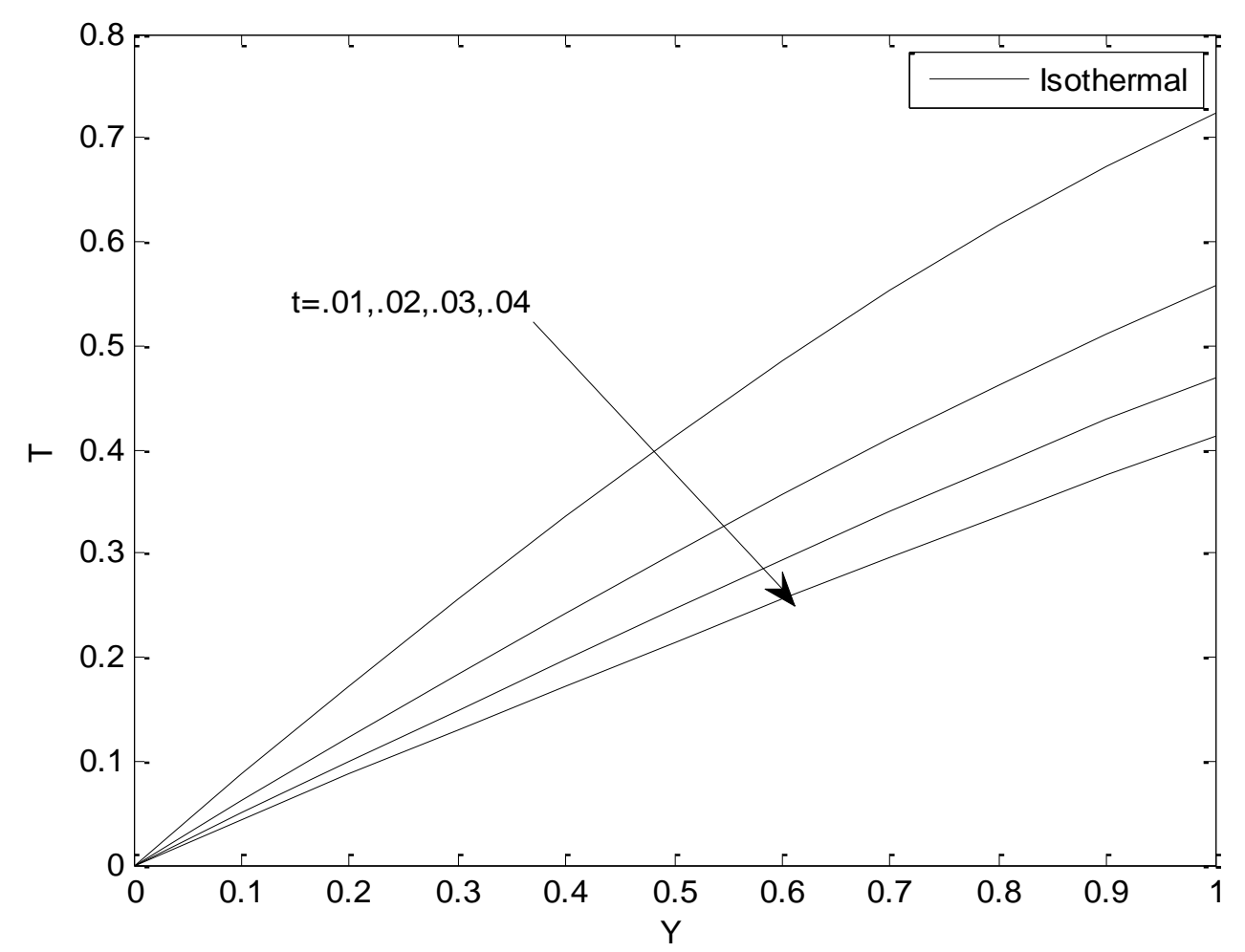

Fig. 12. Temperature field for different values of $t$ in Isothermal plate. when $K^{2}=4, M=2, R=2, K_{1}=0.2, G_{r}=3$

\section{CONCLUSIONS}

The present study investigates the unsteady hydromagnetic free convection boundary layer flow of a viscous incompressible electrically conducting fluid past a ramped temperature impulsively moving plate in a rotating porous medium in the presence of inclined magnetic field and thermal radiation. The significant findings are summarized as:

For both ramped temperature and isothermal plates:

Magnetic field tends to reduce fluid flow in both the ramped and isothermal cases. Increase of radiation rotation decreases the fluid flow in the isothermal plate whereas it increases the fluid flow in the ramped temperature. Rotation, Inclined magnetic field angle and time decrease the fluid flow in both cases. Prandtl number has tendency to increase the fluid temperature. Radiation parameter casus the decrease of temperature in ramped temperature case but it reversed in isothermal case. Here inclination angle causes the increase and decrease of magnetic field effect so at $\theta=\frac{\pi}{2}$ it acts like transverse magnetic field.

\section{REFERENCES}

Arpaci V.S (1968). Effects of thermal radiation on the laminar free convection from a heated vertical plate, Int. J. Heat Mass Transfer, Vol. 11, pp. 871-881.
Bestman A.R. and Adjepong S.K (1988).Unsteady hydromagnetic free-convection flow with radiative heat transfer in a rotating fluid, Astrophysics and Space Science, Vol. 143, pp. 73-80.

Chebbi. B and M. Bouzaiane (2012). On the Effects of Rotation on the Passive Scalar and Kinematic Fields of Homogeneous Sheared Turbulence. Journal of Applied Fluid Mechanics, Vol. 5, No. 2, pp. 55-65, 2012

Jha B.K., A.O. Ajibade (2010). Unsteady free Convective Couette flow of heat Generating/absorbing fluid .Int. J. Energy and Tech., 2(12), 1-9.

Kumar A.G.V., S.V.K Varma (2011). Radiation effects on MHD flow past an impulsively started exponentially accelerated vertical plate with variable temperature in the presence of heat generation .Int. J. Eng. Sci.Tech.,3(4),p 2897.

Narahari M., (2010). Effects of thermal radiation and free convection currents on the Unsteady Couette flow between two vertical parallel plates with constant heat flux WSEAS Transactions on Heat and Mass Transfer, 5(1), 21-30

Rajput U.S.,S. Kumar (2011). Combined effects of rotation and radiation on MHD flow past an impulsively started vertical plate with variable temperature Int. J. Math. Analysis,5(24), 1155 1163. 
Rajput U.S., P.K. Sahu (2011) .Effect of a uniform transverse magnetic field on the unsteady transient free convection flow of an incompressible viscous electrically conducting fluid between two infinite vertical parallel plates with constant temperature Int. J. Math. Analysis,5(34), 1665-6671.

Sandeep and Sugunamma (2013) “Aligned Magnetic Field And Chemical Reaction Effects On Flow Past A Vertical Oscillating Plate Through Porous Medium" Communications in applied sciences, Vol. 1,Number 1,2013,81-105.

Saxena S. S, G.K. Dubey (2011) .Unsteady MHD heat and mass transfer free convection flow of polar fluids past a vertical moving porous plate in a porous medium with heat generation and thermal diffusion Adva. Appl. Sci. Res., 2 (4), 259-278.
Seth G.S.R. Nandkeolyar and Md.S. Ansari (2012).Effects of Hall Current and Rotation on Unsteady MHD Couette Flow in the Presence of anInclined Magnetic Field. Journal of Applied Fluid Mechanics, Vol. 5, No. 2,pp. 67-74,

Vijayalakshmi A.R(2010). Radiation effects on freeconvection flow past an impulsively started vertical plate in a rotating fluid Theoret. Appl. Mech., Vol.37, No.2, pp.79-95, 